\title{
Doppler Ultrasound Screening of the Uterine Arteries as a Predictor for Early Miscarriage
}

\author{
Aseel Ghazi Rifat ${ }^{*}$ i
}

\begin{abstract}
Objectives: So far, there is no concrete consensus on using uterine Doppler measurements for predicting the adverse outcomes in the early stages of pregnancy between 6-14 weeks. Hence, the current study was planned to investigate the role of uterine artery Doppler in predicting the miscarriage in early pregnancy and to evaluate whether uterine artery Doppler indices abnormality is associated with a complicated pregnancy and adverse outcomes.

Materials and Methods: The present cross-sectional prospective study estimated the uterine artery Doppler of right and left uterine arteries and the mean of pulsatility index (PI), resistance index (RI), and systolic/diastolic (SD) ratio was obtained for each patient. The gestational age, at which the Doppler study was performed, was 6-14 weeks for all groups. Finally, the groups of normal and threatened miscarriage were followed up for the outcome up to 20 weeks of gestation.

Results: There was a significant difference in the means of PI, RI, and S/D ratio among the three groups. However, PI was the only parameter which was significantly associated with the outcome of pregnancy. In addition, the cut-off value for PI was 2.64 at $91 \%$ sensitivity and $81 \%$ specificity for predicting miscarriage.

Conclusions: In general, uterine artery Doppler should be introduced as the routine screening test for identifying the women who could have an adverse pregnancy outcome for its effective management.

Keywords: Uterine artery Doppler, Pregnancy outcome prediction, Screening test
\end{abstract}

\section{Introduction}

Bleeding and miscarriage are the most common causes of seeking medical advice in early pregnancy (1) and spontaneous miscarriage is clinically recognized pregnancy loss before the viability (2). Therefore, effective management techniques and prophylactic strategies can be offered to save a life if a screening test is available that can signal upon the likelihood of miscarriage in the near future (1).

Although the definite cause of miscarriage cannot be addressed in most of the cases, when bleeding happens, it could be expected that a vascular injury may be the primary or secondary cause (3).

Normal implantation and placentation are of great importance for achieving a normal pregnancy. The main event during this period is the trophoblastic cell invasion to the inner third of the myometrium and migration through the entire length of maternal spiral arteries. In addition, the vascular remodeling of these high resistance arteries in the maternal-fetal interface results in low resistance and high flow state in the intervillous space (4). This is associated with a concomitant increase in uterine blood flow and perfusion that regulates uterine receptivity and is crucial for the normal pregnancy outcome $(5,6)$. These changes are reflected in uterine artery Doppler studies by a progressive reduction in the uterine arterial blood flow resistance at five weeks after implantation $(4,7)$.

Extensive evidence is present regarding the benefit of uterine artery Doppler ultrasound for assessing uteroplacental perfusion and predicting the future development of pre-eclampsia, fetal growth restriction, placental abruption, and stillbirth in the second trimester $(8,9)$. However, the researchers have not so far reached a concrete consensus about using uterine Doppler measurements for predicting the adverse outcomes in the early stages of pregnancy between 6-14 weeks. Accordingly, the present sought to determine any association between the uterine artery Doppler measurements and the outcome of pregnancy in this early gestational period. These measurements can be utilized to predict or identify women with complicated pregnancies or higher probability of miscarriage if there is such an association.

\section{Objectives associated with. \\ Materials and Methods \\ Study Design and Setting}

To study the role of uterine artery Doppler in predicting the miscarriage in early pregnancy and to evaluate the abnormality with which uterine artery Doppler indices is 
This is a cross-sectional prospective study which was performed in the outpatient Department of Obstetrics and Gynaecology at Azadi Teaching Hospital, Kirkuk, Iraq. All case approach was taken for enrolling the participants (from April 2015 to December 2018) after applying the inclusion and exclusion criteria.

\section{Sample Size}

Considering that the mean difference for the uteroplacental blood flow in abnormal early intrauterine pregnancy and normal pregnancy was 0.4 based on the previous literature $(10,11)$, the required sample size was 28 in each group at $80 \%$ power and $95 \%$ confidence interval according to the following formula.

Total sample size $=\mathrm{C} / \delta 2$

where $\mathrm{C}=7.9$ for $80 \%$ power and $\mu$ and $\sigma$ are population mean and standard deviation, respectively.

Total sample size $=\mathrm{C} / \delta 2^{+2}$

where $\delta=\frac{\mu_{2}-\mu_{1}}{C}, \mathrm{C}=7.9$ for $80 \%$ power and $\mu$ and $\sigma$ denote the population mean and standard deviation, respectively.

\section{Inclusion Criteria}

The sample was drawn from the population of women who had a normal pregnancy, as well as those with threatened miscarriage or diagnosed with missed miscarriage all at 6-14 weeks and calculated from the first day of the last menstrual 88 period with singleton visible intrauterine gestational sac.

\section{Exclusion Criteria}

Women with multiple pregnancies, molar pregnancy, ectopic pregnancy, maternal history of systemic disease and medical co-morbidity, as well as uterine malformation and uterine pathology like myomas or polyps or cervical abnormalities.

Data Collection Tool and Procedure

Totally, 39 women with normal intrauterine pregnancy (group A), 28 women with threatened miscarriage (group B), and 18 women diagnosed as the cases of missed miscarriage (group C) were enrolled in the study following the exclusion criteria for participant selection. A patient record sheet was used to record all the details of the participant, including age, gestational age, parity, and the readings of Doppler indices for right and left uterine arteries. The women having a normal pregnancy and threatened miscarriage were followed till 20 weeks of gestation. At 20 weeks of gestation, the pregnancy outcome was recorded for these women.

All women underwent transabdominal Doppler sonographic examination at the time of presentation by the same examiner so that to reduce the interobserver variability using the ultrasound machine (Medison SonoAce R7 from Samsung) equipped with curvilinear 3.5 $\mathrm{MHz}$ abdominal probe and operated at colour Doppler and pulse wave Doppler mode. Women were placed in a semi-recumbent position and the probe was used to obtain the sagittal section of the uterus and the cervical canal, as well as the internal cervical os. Then, the transducer was gently tilted from side to side with color flow mapping used to identify the uterine arteries, followed by utilizing the pulse wave Doppler until obtaining 3 similar consecutive waveforms. The values for pulsatility index (PI), resistance index (RI), and systolic/ diastolic (S/D) ratio were measured and recorded for right and left uterine arteries and the mean was taken for every woman in all 3 groups (Figure 1A-C).

No definite reference values are present for Doppler indices in early pregnancy due to the paucity of Doppler studies before the eight weeks of gestation because of patients' infrequent referral for scanning in early pregnancy. However, the results of a previous study indicated a linear decrease in the values of PI, RI, and S/D ratio with the progression of pregnancy between 4-6 weeks and 16-32 weeks (8). In addition, no definite cutoff values were reported for abnormal results, for example, some of them regarded the cut-off values of PI and RI above the 95th percentile of the mean for gestational age

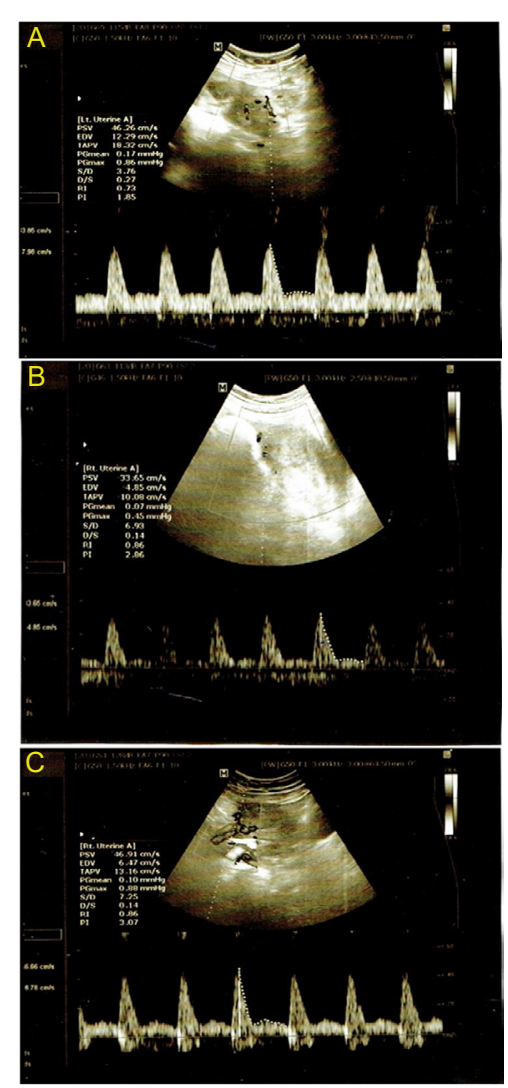

Figure 1. Uterine Artery Doppler Waveform in (A) Normal Pregnancy, (B) Threatened Miscarriage, and (C) Missed Miscarriage. 
as a predictor of the adverse outcome (9).

\section{Statistical Analysis}

Descriptive analysis was used to describe the data in terms of age, gestational age, along with the indices of uterine artery Doppler in terms of mean and standard deviation. For other variables like parity and the pregnancy outcome at 20 weeks, the frequency analysis was performed to describe the data. Furthermore, one-way ANOVA was applied to compare the means of $\mathrm{PI}, \mathrm{RI}$, and $\mathrm{S} / \mathrm{D}$ ratio among the 3 groups. Next, the logistic regression was used to identify the indices from uterine Doppler which were significantly associated with the outcome of interest. This implies that which of the given indices (i.e., RI, PI, or S/D) could precisely predict the pregnancy outcome if the uterine Doppler would be performed in the early pregnancy between 6-14 weeks of gestation. The index which was found to be significantly associated with the outcome was treated with receiver operator characteristic (ROC) curve analysis to determine the cut-off value of that particular index. This cut-off value could predict the index range above which there was a high probability of adverse pregnancy outcome or, in other words, the chances of miscarriage that increased beyond this cut-off value.

\section{Results}

The uterine artery Doppler screening was conducted for both arteries, followed by recording the readings and obtaining the mean for all women.

Table 1 represents the clinical characteristics and the findings of uterine artery Doppler for women in the 3 groups and the total sample. As shown in Table 1, there was no significant difference in the mean age of pregnant women and their gestational age among the 3 groups.

Moreover, no statistically significant difference was observed in the parity among the women in the 3 groups (Table 2).

However, there was a significant difference in the mean values of PI, RI, and S/D ratio among the groups (Table 1). In addition, the mean values of the indices in group $\mathrm{C}$ were significantly higher than those of groups $\mathrm{A}$ and $\mathrm{B}$.

Groups A and B were followed up until the 20 weeks of gestation to find the status of the fetus. In group A, all the pregnant women had a normal pregnancy and there was no report of any adverse outcome. Conversely, five out of 28 pregnancies ended up in adverse outcome in group $B$, all of which demonstrated an increase in the Doppler indices.

Additionally, based on the data in Table 1, the mean values of PI increased from group A (2.0) to group B (2.8) and then, group $C$ (3.2) with a corresponding rise in the adverse outcomes within groups B and C. Hence, the

Table 2. Comparing Parity Among the 3 Groups

\begin{tabular}{|c|c|c|c|}
\hline \multirow{2}{*}{ Groups } & \multicolumn{2}{|c|}{ Parity } & \multirow{2}{*}{ Total } \\
\hline & $0-2$ & $>2$ & \\
\hline Group A & 25 & 14 & 39 \\
\hline Group B & 17 & 11 & 28 \\
\hline Group C & 11 & 7 & 18 \\
\hline Total & 53 & 32 & 85 \\
\hline
\end{tabular}

$P$ value $=0.954$.

Table 1. Clinical and Uterine Doppler Characteristics Among 3 Groups by One-Way ANOVA

\begin{tabular}{|c|c|c|c|c|c|c|c|}
\hline \multirow{2}{*}{ Characteristics } & \multirow{2}{*}{ Group } & \multirow{2}{*}{$\mathbf{N}$} & \multirow{2}{*}{ Mean \pm SD } & \multirow{2}{*}{ Overall } & \multicolumn{3}{|c|}{$P$ Value* } \\
\hline & & & & & Group A vs. B & Group B vs. C & Group A vs. C \\
\hline \multirow{4}{*}{ Age (y) } & Group A & 39 & $28.9 \pm 4.9$ & \multirow{4}{*}{0.075} & \multirow{4}{*}{0.275} & \multirow{4}{*}{0.035} & \multirow{4}{*}{0.173} \\
\hline & Group B & 28 & $26.1 \pm 5.7$ & & & & \\
\hline & Group C & 18 & $27.6 \pm 3.9$ & & & & \\
\hline & Total & 85 & $27.7 \pm 5.1$ & & & & \\
\hline \multirow{4}{*}{ Gestational age (wk) } & Group A & 39 & $9.1 \pm 2.5$ & \multirow{4}{*}{0.908} & \multirow{4}{*}{0.746} & \multirow{4}{*}{0.995} & \multirow{4}{*}{0.766} \\
\hline & Group B & 28 & $9 \pm 2.6$ & & & & \\
\hline & Group C & 18 & $9.3 \pm 2.5$ & & & & \\
\hline & Total & 85 & $9.1 \pm 2.5$ & & & & \\
\hline \multirow{4}{*}{ Pulsatility index } & Group A & 39 & $2 \pm 0.4$ & \multirow{4}{*}{$<0.001$} & \multirow{4}{*}{0.068} & \multirow{4}{*}{0.913} & \multirow{4}{*}{0.085} \\
\hline & Group B & 28 & $2.8 \pm 0.6$ & & & & \\
\hline & Group C & 18 & $3.2 \pm 0.5$ & & & & \\
\hline & Total & 85 & $2.5 \pm 0.7$ & & & & \\
\hline \multirow{4}{*}{ Resistance index } & Group A & 39 & $0.7 \pm 0.1$ & \multirow{4}{*}{$<0.001$} & \multirow{4}{*}{0.389} & \multirow{4}{*}{0.894} & \multirow{4}{*}{0.4} \\
\hline & Group B & 28 & $0.7 \pm 0.1$ & & & & \\
\hline & Group C & 18 & $0.9 \pm 0.1$ & & & & \\
\hline & Total & 85 & $0.7 \pm 0.2$ & & & & \\
\hline \multirow{4}{*}{ Systolic/diastolic ratio } & Group A & 39 & $5.9 \pm 2.6$ & \multirow{4}{*}{$<0.001$} & \multirow{4}{*}{0.017} & \multirow{4}{*}{$<0.001$} & \multirow{4}{*}{$<0.001$} \\
\hline & Group B & 28 & $5.7 \pm 2$ & & & & \\
\hline & Group C & 18 & $9.3 \pm 0.8$ & & & & \\
\hline & Total & 85 & $6.5 \pm 2.6$ & & & & \\
\hline
\end{tabular}

Note. ${ }^{*} P$-values in bold denote significant $P$ values of less than 0.05 at a $95 \%$ confidence interval, SD: Standard deviation; ANOVA: Analysis of variance. 
Table 3. Logistic Regression

\begin{tabular}{lccccc}
\hline Doppler Indices & B & Standard Error & Wald & df & P \\
\hline S/D & 0.184 & 0.595 & 0.095 & 1 & 0.758 \\
RI & -5.108 & 5.966 & 0.733 & 1 & 0.392 \\
PI & 3.601 & 1.394 & 6.67 & 1 & 0.01 \\
Constant & -11.438 & 4.595 & 6.196 & 1 & 0.006 \\
\hline
\end{tabular}

S/D, systolic/diastolic; standard deviation; RI: resistance index; PI: pulsatility index.

results indicate that the uterine Doppler measurements can probably predict the adverse outcome thus the researchers proceeded with the logistic regression to explore which of the Doppler measurements was significantly related to the outcome of interest.

Table 3 presents the logistic regression on a complete sample of 85 pregnant women (group $\mathrm{A}+$ group $\mathrm{B}+$ group C) by taking the outcome of pregnancy on Y-axis, along with the uterine Doppler readings on $\mathrm{X}$-axis as independent variables. The results revealed that only PI, among the 3 Doppler indices, was significantly related to the precise adverse outcome at 20 weeks.

The $\operatorname{Exp}$ (B) for PI was equal to 36.63 which represented that the odds of having an adverse pregnancy outcome or miscarriage was 36.63 times higher among women who had a mean PI value of 3.2 .

In addition, the receiver operator characteristic curve was plotted (Figure 2) to determine the cut-off value for PI above which the probability of the adverse outcome could be predicted accordingly.

Based on this graph, the area under the curve was 0.919 with a standard error of 0.03 (95\% CI: 0.86-0.98), which implied that the PI could perfectly predict the occurrence of the adverse outcome among pregnant women. Similarly, the cut-off value for PI was 2.64 at $91 \%$ sensitivity and $81 \%$ specificity for miscarriage, indicating that one can

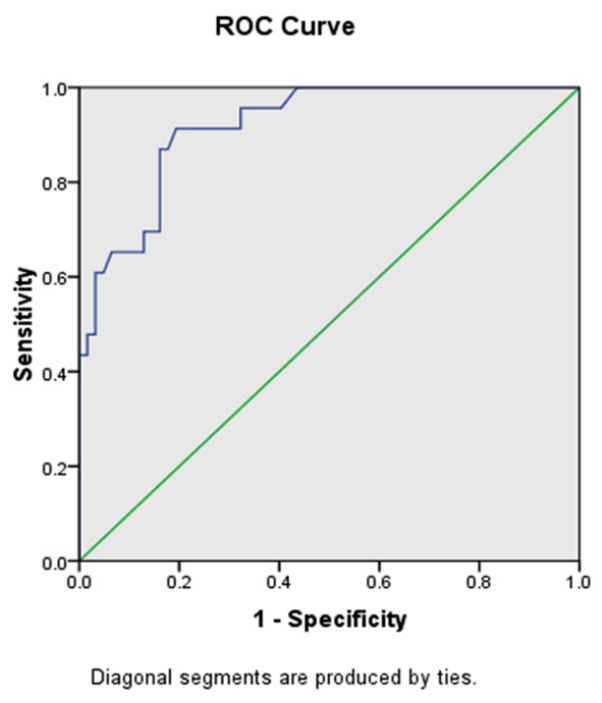

Figure 2. Receiver Operator Characteristic Curve Showing Cutoff Value for Pulsatility Index. expect an adverse pregnancy outcome by using the uterine Doppler investigation at an early stage if the value of PI crosses 2.64.

\section{Discussion}

Miscarriage is regarded as a common complication in the first half of pregnancy since up to $50 \%$ of conceptions fail to pass beyond 20 weeks of gestation (12). The main aim of antenatal care is to determine the vulnerable fetus in order to provide the clinical interferences that may lead to a reduction in perinatal mortality and morbidity $(8,13)$.

The uteroplacental circulation is the net product of the trophoblastic cell invasion to the wall of the spiral arteries that occurs in 2 waves. The initial wave of the vascular invasion initiates at the 4th-5th weeks of gestation, followed by a subsequent wave of invasion at 14th week, and then low-resistance vessels are identified at the site of future placenta approximately four weeks after the implantation (14). These changes result in a progressive increase in the uterine blood supply and the initiation of the uteroplacental circulation with the characteristics of high volume and extensive diastolic element (15). However, pregnancy causes no significant difference in the flow of the main uterine branches until establishing a well-developed intervillous circulation (16).

Early completion of the 2 waves of invasion and diagnosis of the missed miscarriage are difficult thus the present study was performed at the gestational age 6-14 weeks.

Doppler ultrasound is becoming beneficial noninvasive equipment for the evaluation of fetal well-being and the placental circulation in the second and third trimesters of pregnancy in addition to providing valuable data upon the uteroplacental circulation physiology (15). Moreover, various studies showed that the blood flow resistance of the uterine arteries in the midluteal phase of non-pregnant cycles elevates in women with repeated pregnancy loss compared to normal women $(6,18-19)$. Likewise, the results of another study indicated that uterine artery Doppler assessment may be beneficial for choosing the management modalities in patients with the first-trimester miscarriage (1).

The vessels that provide the uterine blood supply have extensive anastomoses leading to even violence in the bloodstream of the uterine artery (12). In the present study, Doppler measurements were performed on the uterine arteries because these vessels are easily 
detected and identified which makes the results more reproducible. On the other hand, the smaller vessels like the peritrophoblastic vessels are difficult to identify and need more sophisticated devices for detection; this makes the measurements imprecise (16).

Our results revealed a significant difference in the 3 Doppler indices between the studied groups with group $\mathrm{C}$ showing the highest significant mean values. These findings suggest that there might be a subclinical vasculopathy impairing the uterine blood supply and, subsequently, causing the early pregnancy failure in group C. In addition, angiogenesis and the blood supply to the uterus are fundamental for endometrial growth and embryonic development (3).

Studies assessing the role of uterine artery Doppler changes in women with miscarriage obtained conflicting results, for example, some of them found that alterations in Doppler indices in one or both uterine arteries were associated with miscarriage (3,14,20-22). Contrarily, other studies $(10,16,23)$ reported that the use of the Doppler ultrasound is unhelpful in the prediction of the outcome in early pregnancy and indicated that the vascular compromise in the uteroplacental circulation in abnormal pregnancies was extremely minimal to be detected by the Doppler ultrasound. Their different findings may be attributed to the heterogeneity of the etiologies of the miscarriage and to the fact that they used different study groups (16), including only patients with threatened miscarriage and excluding the cases of missed miscarriage at a narrower range of gestational age $(10,23)$.

In addition to providing information on Doppler indices for each group of pregnant women, the current study guided us to identify the index which could predict the future outcome of the pregnancy. In other words, it revealed that only the mean value of the PI was significantly related to the outcome of pregnancy. This finding is in line with the finding of El Behery et al (3) that represented a link between the unilateral elevation of uterine arteries $\mathrm{PI}$ in the first trimester and the subsequent occurrence of miscarriage. However, Romero-Gutiérrez et al found that increased RI was the parameter that was associated with the adverse pregnancy outcome (21).

Furthermore, the results of this study revealed that the cut-off value for the PI of 2.64 was highly sensitive and specific for predicting the miscarriage. Additionally, Wahab et al (6) in their study found that the aborted patients had uterine artery PI more than 2.5 while those who attained term had the uterine artery PI value of 2.5 or less. However, the researchers could not predict the outcome of pregnancy depending on this cut-off value due to the small number of patients who became pregnant and reached term in their study. This was the case with the other study that also failed to provide the cut-off for the indices since it was a cross-sectional study which was conducted among the high-risk pregnancies and lacked a control group and the follow-up to evaluate the pregnancy outcome of the enrolled women (14).

To the best of our knowledge, this is the first study that has focused on the early pregnancy period with a mean gestational age of nine weeks regarding identifying the best index and its cut-off value to be used as the screening test for early pregnancy failure.

However, the small sample size of each group and the inability to address the confounders are considered as the limitations of this study. Therefore, other researchers are recommended to design further longitudinal studies with a larger sample size including these 3 groups of women from the cohort of the pregnant population. In addition, such studies are suggested to take into consideration confounders like the weight and height of individuals, dietary pattern, as well as whether the women are seeking antenatal care at timely intervals and by qualified health personnel, and the like so that to explore the real association between the indices and the pregnancy outcome.

\section{Conclusions}

Overall, uterine artery Doppler helps predict the miscarriage in early pregnancy. Accordingly, it should be introduced as a routine screening test for identifying women who are at the risk of developing miscarriage in order to offer effective prophylactic management strategies such as rest, aspirin, heparin, progestogenic supplementation, and the like.

\section{Conflict of Interests}

The authors declare that they have no conflicts of interest.

\section{Ethical Issues}

The permission to conduct this study was taken from the Institute Review Board and informed consent was obtained from each participant before starting the procedure. The participants were free to discontinue the study at any time. Further, they were informed about the benefits of the study and were assured of the confidentiality of their information. The study was approved by the Ethical Committee of Kirkuk University with approval number 342.

\section{Financial Support}

The study received no funding from any source.

\section{References}

1. Guedes-Martins L, Saraiva JP, Gaio AR, Reynolds A, Macedo F, Almeida H. Uterine artery Doppler in the management of early pregnancy loss: a prospective, longitudinal study. BMC Pregnancy Childbirth. 2015;15:28. doi:10.1186/ s12884-015-0464-9

2. Hoffman BL, Schorge JO, Schaffer JI, et al. First-Trimester Abortion. Williams Gynecology; 2008.

3. El Behery MM, Siam S, Seksaka MA, Mansour SM. Uterine artery Doppler and urinary hyperglycosylated HCG as predictors of threatened abortion outcome. 
Middle East Fertil Soc J. 2013;18(1):42-46. doi:10.1016/j. mefs.2012.10.001

4. Lin S, Shimizu I, Suehara N, Nakayama M, Aono T. Uterine artery Doppler velocimetry in relation to trophoblast migration into the myometrium of the placental bed. Obstet Gynecol. 1995;85(5 Pt 1):760-765. doi:10.1016/00297844(95)00020-r

5. Trayanov I, Dimitrakova E. Doppler velocimetry of the uterine arteries: an early screening test for miscarriage. Pril (Makedon Akad Nauk Umet Odd Med Nauki). 2016;37(1):51-56. doi:10.1515/prilozi-2016-0007

6. Wahab HA, El-Din DS, Zain E, Abdelgany M, Youssef MAFM. Uterine artery Doppler and subendometrial blood flow in patients with unexplained recurrent miscarriage. Middle East Fertil Soc J. 2011;16(3):209-214. doi:10.1016/j. mefs.2011.04.001

7. Nakatsuka M, Habara T, Noguchi S, Konishi H, Kudo T. Impaired uterine arterial blood flow in pregnant women with recurrent pregnancy loss. J Ultrasound Med. 2003;22(1):27-31. doi:10.7863/jum.2003.22.1.27

8. Dickey RP. Doppler ultrasound investigation of uterine and ovarian blood flow in infertility and early pregnancy. Hum Reprod Update. 1997;3(5):467-503. doi:10.1093/ humupd/3.5.467

9. Khong SL, Kane SC, Brennecke SP, da Silva Costa F. First-trimester uterine artery Doppler analysis in the prediction of later pregnancy complications. Dis Markers. 2015;2015:679730. doi:10.1155/2015/679730

10. Li H, Gudmundsson S, Olofsson P. Uterine artery blood flow velocity waveforms during uterine contractions. Ultrasound Obstet Gynecol. 2003;22(6):578-585. doi:10.1002/uog.921

11. Hofstaetter C, Dubiel M, Gudmundsson S, Marsal K. Uterine artery color Doppler assisted velocimetry and perinatal outcome. Acta Obstet Gynecol Scand. 1996;75(7):612-619. doi:10.3109/00016349609054684

12. Trayanov I, Dimitrakova E. Doppler velocimetry of the uterine arteries: an early screening test for miscarriage. Pril (Makedon Akad Nauk Umet Odd Med Nauki). 2016;37(1):51-56. doi:10.1515/prilozi-2016-0007

13. Gómez $\mathrm{O}$, Martínez JM, Figueras $\mathrm{F}$, et al. Uterine artery Doppler at 11-14 weeks of gestation to screen for hypertensive disorders and associated complications in an unselected population. Ultrasound Obstet Gynecol.
2005;26(5):490-494. doi:10.1002/uog.1976

14. Mahmoud M. Predictive value of doppler examination of uteroplacental circulation in early pregnancy and serum B-HCG in its adverse outcome. Al-Azhar Assiut Med J. 2015;13(3):43-49.

15. Jaffe R, Warsof SL. Color Doppler imaging in the assessment of uteroplacental blood flow in abnormal first trimester intrauterine pregnancies. An attempt to define etiologic mechanisms. J Ultrasound Med. 1992;11(1):41-44. doi:10.7863/jum.1992.11.1.41

16. Pellizzari P, Pozzan C, Marchiori S, Zen T, Gangemi M. Assessment of uterine artery blood flow in normal firsttrimester pregnancies and in those complicated by uterine bleeding. Ultrasound Obstet Gynecol. 2002;19(4):366-370. doi:10.1046/j.1469-0705.2002.00667.x

17. Dave A, Joshi R, Sooruthiya S, Dave A. Role of uterine artery Doppler in prediction of FGR in high risk pregnancies in 20-24 weeks. nt J Reprod Contracept Obstet Gynecol. 2017;6(4):1388-1391. doi:10.18203/2320-1770. ijrcog20171396

18. Abdel Razik M, Goher M, Shedid A, Idris T. Doppler study of uterine arteries blood flow in patients with unexplained recurrent pregnancy loss and the effects of nitric oxide donors. Med J Cairo Univ. 2012;80(1):421-425.

19. Habara T, Nakatsuka M, Konishi H, Asagiri K, Noguchi S, Kudo T. Elevated blood flow resistance in uterine arteries of women with unexplained recurrent pregnancy loss. Hum Reprod. 2002;17(1):190-194. doi:10.1093/humrep/17.1.190

20. Al-Waeely FA. Evaluation of doppler ultrasound study in first trimester treatened abortion and anembryonic pregnancy. Med J Basrah Univ. 2005;23(2):5-9.

21. Romero-Gutiérrez G, Huebe-Martínez AA, AmaralNavarro I, Ruiz-Treviño AS. Doppler ultrasound assessment in women with threatened abortion. Clin Med Res. 2013;2(3):24-28. doi:10.11648/j.cmr.20130203.11

22. Nakatsuka M, Habara T, Noguchi S, Konishi H, Kudo T. Impaired uterine arterial blood flow in pregnant women with recurrent pregnancy loss. J Ultrasound Med. 2003;22(1):27-31. doi:10.7863/jum.2003.22.1.27

23. Alcázar JL, Ruiz-Perez ML. Uteroplacental circulation in patients with first-trimester threatened abortion. Fertil Steril. 2000;73(1):130-135. doi:10.1016/s00150282(99)00486-0

(C) 2020 The Author (s); This is an open-access article distributed under the terms of the Creative Commons Attribution License (http://creativecommons.org/licenses/by/4.0), which permits unrestricted use, distribution, and reproduction in any medium, provided the original work is properly cited. 